\title{
Theoretical Implications of the Elastic Modulus Discontinuity in Rubber Networks
}

\author{
DAVID E. HANSON, ${ }^{1}$ C. M. ROLAND ${ }^{2}$ \\ ${ }^{1}$ Theoretical Division, Los Alamos National Laboratory, Los Alamos, New Mexico 87545 \\ ${ }^{2}$ Chemistry Division, Naval Research Laboratory, Washington, DC 20375-5342
}

Received 4 January 2010; revised 23 February 2010; accepted 13 April 2010

DOI: $10.1002 /$ polb.22045

Published online in Wiley InterScience (www.interscience.wiley.com).

\begin{abstract}
The existence of a discontinuity in the modulus of rubber as the strain transitions from compression to extension is strongly suggested by multiple experiments. Classical rubber elasticity theories, however, do not admit such behavior. Here, we investigate a modification of the assumptions of classical elasticity theory to reconcile this discrepancy. We present an analysis of the consequences of assuming that chain forces are nonzero only for chain extension relative to the unstrained state, in contrast to the classical elasticity theory, which assumes that the chain force is directly proportional to the chain end-to-end distance (an entropic spring). Assuming an affine transformation of the network node coordinates, we
\end{abstract}

derive two modulus discontinuity factors between compression and extension: $D_{1}$, based on the differing number of network chains being extended and $D_{2}$, based on the average differential chain extension. The discontinuities arise due to geometric effects, inherent in the affine transformation between compressive and extensive strains. We find that $D_{1}$, the ratio of the numbers of participating chains (compressive/extensive $=1.37$ ), suffices to account for the experimentally observed modulus discontinuity in natural rubber of 1.34. (c) 2010 Wiley Periodicals, Inc. J Polym Sci Part B: Polym Phys 48: 1795-1798, 2010

KEYWORDS: elastomers; strain; stress; theory

\section{BACKGROUND}

Classical models for rubber elasticity assume that the elastic response of a rubber network is due to the tendency of the individual chains to seek conformations with the most probable end-to-end distances, $R$. For idealized chains, in one dimension a Markov walk analysis shows that the end-toend distance distribution is Gaussian, with its peak at $R=0$. Hence, the most probable end-to-end distance is also zero. For chains free to move in three dimensions, the distribution peaks at a finite value of $R$, due to the added weighting factor of the surface area of the sphere of radius $R$, which constrains one end of the chain. However, for a chain in a rubber network, having both ends fixed to crosslink nodes, the first condition is thought to apply. The equilibrium for network chains is their configuration at the moment of network formation, which would be the three-dimensional value of $R$ $>0$. The entropy $S$, is taken as the derivative of the end-toend distance distribution function, and the change in free energy is given by $T d S$. For a chain connecting two network nodes, the retractive force, assumed to act along the vector defined by the connected nodes, is given $b^{1}$ :

$$
\vec{F}_{\text {chain }}=\frac{3 k T}{N b^{2}} \vec{R}
$$

where $k$ is Boltzman's constant, $T$ is the absolute temperature, $N$ is the number of uncorrelated beads on the chain, each of length $b$, and $R$ is the end-to-end distance. This force is commonly referred to as the 'entropic spring' as it is proportional to $R$.

Numerous network models, based on this chain force, have been developed over the past 70 years. These models usually stipulate uniform chain lengths, chain orientations that are symmetric with respect to the strain axis and enforce an affine transformation of the node coordinates in response to compressive or tensile strains. The simplest of these affine network models, due to Kuhn, ${ }^{2}$ relates the engineering stress to the extension factor $\lambda$ as:

$$
\sigma_{\mathrm{eng}}=G\left(\lambda-1 / \lambda^{2}\right)
$$

where $G$ is the shear modulus. This expression is commonly referred to as the "reduced force" formula. Although the model is known to disagree with experiment at moderate and high extensions, it is considered to be accurate for small extensions. The second term in eq 2 , which results from the assumption of incompressibility, is due to the idealized network chains that are initially aligned perpendicular to the strain axis. Under an affine transformation, their end-to-end distances decrease and, because the chain force is assumed to be a perfect spring, these chains tend to assist the applied stress, that is, tend to 'push' the network along the strain axis.

The modulus, defined as the derivative of the stress with respect to the strain $(\lambda-1)$, may be obtained by 
differentiating eq 2 . It is clearly continuous at $\lambda=1$. The Kuhn model assumes affine behavior; however, the opposite assumption, referred to as phantom chains ${ }^{3-5}$ similarly predicts continuity of the modulus at $\lambda=1$. To our knowledge, all rubber elasticity theories predict that the modulus is continuous at zero strain. Experiments on both poly(dimethylsiloxane $)^{6,7}$ and natural rubber $^{8-13}$ however, strongly suggest that the modulus is discontinuous at zero strain.

It is likely that some modification of the classical elasticity theories is needed to reconcile these experiments. Here, we will examine the consequences of assuming that network chain forces are positive and nonzero only for extensions beyond the initial end-to-end distance. That is, we shall assume that chains whose end-to-end distance decreases in response to an affine transformation of the network nodes do not contribute to the elastic stress. We shall refer to this as the "Relaxed Chain Assumption." This represents a significant departure from the classical theories.

\section{GEOMETRIC PROPERTIES OF THE AFFINE TRANSFORMATION}

In cylindrical coordinates, an arbitrary network chain connecting two crosslinks is commonly represented by its endto-end vector $R$, with one end assumed to be at the origin, and having an initial length, $R_{0}$. The vector $R$ may be decomposed into two orthogonal projections: $z$, along the strain axis and $r$, perpendicular to the strain axis:

$$
\begin{aligned}
& z=R_{0} \cos \theta \\
& r=R_{0} \sin \theta,
\end{aligned}
$$

where $\theta$ is the angle between $R$ and the strain axis. For an extension factor of $\lambda$, an affine transformation of the network node coordinates is defined as:

$$
\begin{aligned}
& z^{\prime}=\lambda z \\
& r^{\prime}=r / \sqrt{\lambda}
\end{aligned}
$$

The end-to-end distance transforms as:

$$
R^{2}(\lambda)=\lambda^{2} z^{2}+r^{2} / \lambda
$$

We define a chain extension factor for an arbitrary chain as:

$$
E^{2}(\lambda)=R^{2}(\lambda) / R_{0}^{2}=\lambda^{2} \cos ^{2} \theta+\sin ^{2} \theta / \lambda .
$$

Depending on the angle, the chain extension may be either greater or less than one. At some critical angle $\theta_{c}$, the chain extension is exactly 1 ; the chain vector undergoes a pure rotation. We can calculate $\theta_{\mathrm{c}}$ at $\lambda=1$ by first solving eq 6 for $E$, then taking its derivative with respect to $\lambda$.

$$
\begin{gathered}
E(\lambda, \theta)=\left[\lambda^{2} \cos ^{2} \theta+\sin ^{2} \theta / \lambda\right]^{1 / 2}, \\
\frac{\partial E}{\partial \lambda}=\frac{1}{2}\left[2 \lambda \cos ^{2} \theta-\sin ^{2} \theta / \lambda^{2}\right]\left[\lambda^{2} \cos ^{2} \theta+\sin ^{2} \theta / \lambda\right]^{-1 / 2} .
\end{gathered}
$$

We compute the critical angle by setting the derivative of the chain extension factor to zero, and solving for $\theta$, which may be evaluated at $\lambda=1$.

$$
\begin{gathered}
\lambda \cos ^{2} \theta-\frac{1}{2 \lambda^{2}} \sin ^{2} \theta=0 . \\
\theta_{c}=\tan ^{-1}\left(\sqrt{2 \lambda^{3}}\right)=0.9553 \mathrm{rad} .
\end{gathered}
$$

For extension $(\lambda>1)$, only those network chains making an angle $<\theta_{\text {c }}$ contribute to the elastic force. Compression $(\lambda<$ 1) may be thought of as $2 \mathrm{D}$ expansion and, for this case, only chains making an angle $>\theta_{c}$ contribute. The number of participating chains is not the same for the two cases. If we assume that all orientations of network chains are equally likely and that all chains have been translated to share a common origin, we can calculate the fraction of chains that are stretched during extension by integrating the fraction of solid angle subtended by chains having angles $<\theta_{\text {c. }}$.

$$
f_{\text {tension }}=\int_{0}^{\theta_{c}} \sin \theta d \theta=0.42271 \text {. }
$$

The analogous factor for compressive strains, $f_{\text {compression }}$ can be obtained by performing the integral in eq 11 between $\theta_{c}$ and $\pi / 2$, or simply as $1-f_{\text {tension }}=0.57729$. We define our first modulus discontinuity factor, $D_{1}$, as $f_{\text {compression }} / f_{\text {tension }}=$ 1.366. Note that, in calculating $D_{1}$, we have made no assumption about either the direction or form of the chain forces, only that the stress is proportional to the number of chains stretching.

Our second modulus discontinuity factor, $D_{2}$, is motivated by the angular dependence of the chain extension factor defined by eq 6 . As is clear from eqs 3 and 5, under an affine transformation, the change in the chain end-to-end distance, and hence the average chain force must depend on the initial angle that the chain end-to-end vector makes with the strain axis. In general, one would not expect that the average chain extension factors (and forces) to be the same in compression and tension, and this would also contribute to a discontinuity in the modulus at zero strain. To calculate average chain extension factors, we make the simplest assumption about the form of the force function, that it is a linear spring (spring constant $c$ ), analogous to classical elasticity theory. However, unlike classical elasticity theory, we shall assume that the displacement variable is defined as the change in the end-to-end distance with respect to the initial end-to-end distance, $R_{0}$, not zero. With the additional requirement that the force be zero for negative displacements, the chain force is defined as

$$
\begin{aligned}
& \vec{F}_{\text {chain }}=c \vec{r}, \quad r>0 \\
& \vec{F}_{\text {chain }}=0, \quad r \leq 0,
\end{aligned}
$$

where $r=R-R_{0}$ and the direction of the force is along the chain end-to-end vector. To calculate these average forces, we integrate the chain extension, given by eq 8, over the appropriate solid angle limits. As we are interested in the modulus at zero strain, we may set the extension factor $\lambda$ 


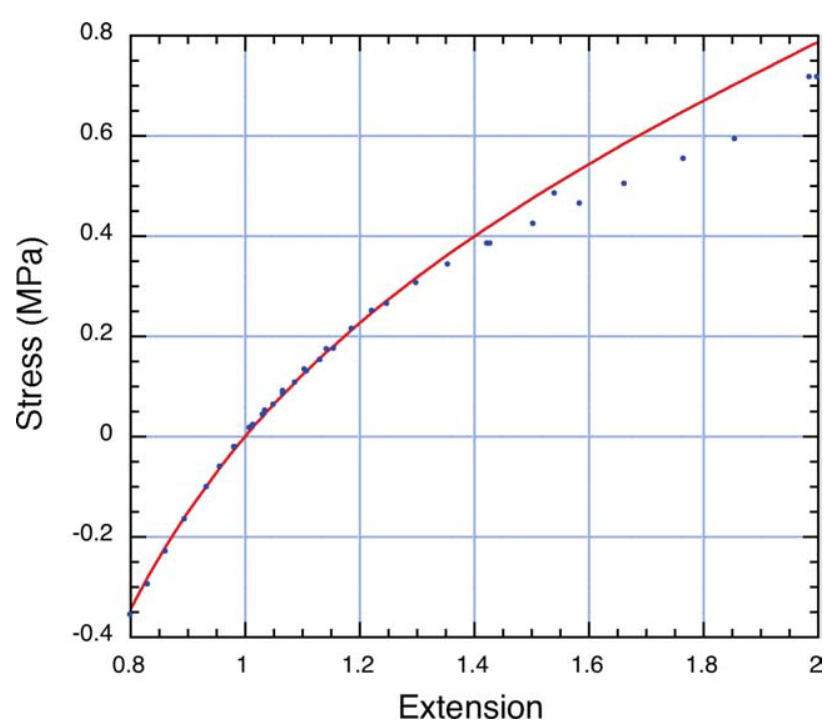

FIGURE 1 Engineering stress vs. strain for natural rubber (Mott and Roland, 1996, blue dots) and fit of eq 2 ( $G=0.45 \mathrm{MPa}$, red line).

to 1 . With this substitution in eq 8 , we define the angledependent chain extension factor as:

$$
G(\theta)=\frac{1}{2}\left[2 \cos ^{2} \theta-\sin ^{2} \theta\right] .
$$

Then the average chain extension (and force) for a sample undergoing tensile strain is given by

$$
g_{\text {tension }}=\frac{1}{\theta_{2}-\theta_{1}} \int_{\theta_{1}}^{\theta_{2}} G(\theta) \sin \theta d \theta
$$

Expanding the expression for $G(\theta)$, we have

$$
g_{\text {tension }}=\frac{1}{\theta_{2}-\theta_{1}} \int_{\theta_{1}}^{\theta_{2}}\left(\cos ^{2} \theta-\frac{1}{2} \sin ^{2} \theta\right) \sin \theta d \theta,
$$

with limits of integration of zero to $\theta_{\mathrm{c}^{\text {. }}}$. An analytic solution for eq 15 can be found in most tables of integrals [Dwight]

$$
g_{\text {tension }}=\frac{1}{\theta_{2}-\theta_{1}}\left[-\frac{1}{3} \cos ^{3} \theta+\frac{1}{2}\left(\frac{1}{3} \cos ^{3} \theta-\cos \theta\right)\right]_{\theta 1}^{\theta_{2}} \text {. }
$$

Using the value for $\theta_{c}$, obtained in eq 10 , we calculate $g_{\text {tension }}$ to be 0.2015 . The average chain extension for compressive strains is also obtained from eq 16 , with integration limits of $\phi_{\mathrm{c}}$ and $\pi / 2 ; g_{\text {compression }}=0.3127$. We define the second modulus discontinuity factor, $D_{2}$, as the ratio of the average chain extension factors, $g_{\text {compression }} / g_{\text {tension }}=1.552$. If our assumption that the retractive force of a chain is directed along its end-to-end vector is correct, then we would expect the modulus discontinuity to be the product of the two factors, $D_{1} \times D_{2}=2.12$.

\section{COMPARISON WITH EXPERIMENT}

The measurements of Mott and Roland ${ }^{9}$ provide well characterized experimental data for comparison. Using natural rubber (cis-1,4-polyisoprene) with two parts per hundred dicumylperoxide (corresponding to a stoichiometric crosslink density of $66 \mathrm{~mol} / \mathrm{m}^{3}$, assuming tetra-functional crosslinks), they measured the stress, in both compression and extension, over a wide range of strains at very low strain rate, $10^{-6} \mathrm{~s}^{-1}$. By gluing the ends of cylindrical samples to metal plates, they were able to measure stress versus strain, in both compression and extension, on the same sample. Their data, over a wide range of extensions is shown in Figure 1. To verify the absence of stress hysteresis, they alternately increased and decreased the applied load. Their data clearly show that a significant discontinuity exists in the modulus at zero strain. Figure 2 shows the data in the vicinity of zero strain and least squares linear fits for each side. The point of zero strain was determined by extrapolating the stress to zero from the compressive side and this results in a slight discontinuity $(\sim 10 \mathrm{kPa})$ for the stress extrapolated to zero strain from the tensile side. We believe that the stress discontinuity is likely an experimental artifact. From the slopes of the linear fits (1.566 and $1.173 \mathrm{MPa}$, respectively, for compression and tension), the calculated ratio of the compressive modulus to the tensile modulus is 1.340 , which is very close to our value for $D_{1}, 1.366$. Clearly, the first discontinuity factor achieves agreement with experiment, leaving us with the question of why the second discontinuity factor is unnecessary. $D_{2}$ required two additional assumptions: (1) that the force was proportional to the end-to-end distance extension factor and, (2) that the force was directed along the chain end-to-end vector. If the retractive force is directed along the chain tangent at the point where is connects to the crosslink node, then the direction of the forces would tend to be random.

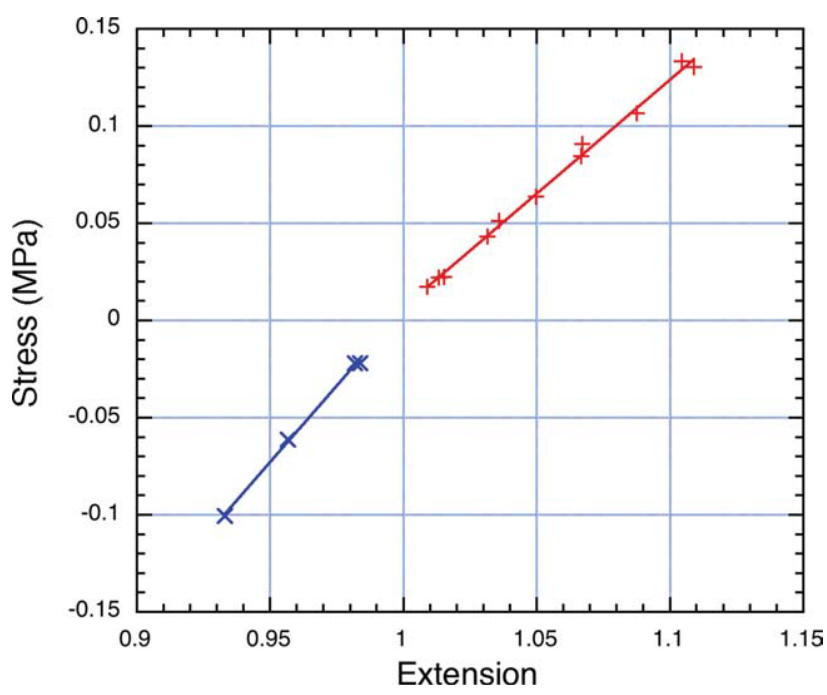

FIGURE 2 Data from Fig. 1 near zero strain and least squares fits for compression (blue $\mathrm{x}$ ) and for extension (red + ). The ratio of the slopes is 1.34 . 
Neither Erman and Flory ${ }^{6}$ nor Pak and Flory ${ }^{7}$ provide suitable data for comparison due to an insufficient number of data points and/or too much scatter in the vicinity of $\lambda=1$. However, both show that the reduced stress (and modulus) seems to be smaller in tension than compression. McKenna and Zapas $^{8}$ reported a smaller modulus difference $(\sim 4 \%)$; however, they found that the magnitude of the discrepancy increased as the strain approached $\lambda=1$.

As shown in Figure 1, classical rubber elasticity theory (eq 2) can be fit reasonably well to our experimental data, in the vicinity of zero strain, by choosing the value of $G$ to be 0.45 $\mathrm{MPa}$. Assuming one tetra-functional crosslink per dicumylperoxide molecule, this modulus would correspond to a chain density about $36 \%$ greater than the stoichiometric limit. So the fit of eq 2 to the data is inconsistent with the crosslinker concentration. Although the reduced force expression (eq 2) is continuous through zero strain, it can exhibit an anomalous modulus discontinuity if linear fits are made for strain regions separated by a significant gap (as is the case for our data). If we make linear fits for the values of eq 2 at the same set of extension factors as the experimental data used in Figure 2, we obtain a stress discontinuity factor of 1.21 . This is significantly less than the value obtained by linear fits to the experimental stress. We do not believe that the observed modulus discontinuity can simply be attributed to the strain gap between the two fit regions.

\section{CONCLUSIONS}

The experimental record strongly suggests that there is a discontinuity in the elastic modulus in the vicinity of zero strain. We have provided a possible explanation of this effect for affine strains by modifying the assumptions of classic rubber elasticity theory (the Relaxed Chain Assumption). Based on the geometric properties of the affine transformation of network node coordinates, we obtained two discontinuity factors for the modulus of rubber at zero strain, $D_{1}$ and $D_{2}$. The assumptions that we make about the chain forces represent a significant departure from conventional rubber elasticity theory. For both factors, we assumed only that the macroscopic stress (and modulus) was proportional to the number of chains being extended. The first factor is a consequence of the differing number of network chains that undergo extension depending on the type of strain imposed on the network, compressive or tensile; more chains are stretched for compressive strain than for tensile strain. We made no assumption about the direction or form of the network chain forces in its derivation. $D_{1}$ is defined as the ratio of the numbers of chains being extended (compressive/ extension) and its value is 1.366 . This is very close to the experimental value for the modulus discontinuity in natural rubber, $1.34 .^{9}$

The second modulus discontinuity factor, $D_{2}$, is defined as the ratio of the average differential stretch of network chains undergoing either compressive or tensile strain. For this factor, we assumed that: (1) the chain forces are proportional to chain extensions with respect to the initial end-to-end distance, (2) the chain force is zero for end-to-end distances less than the initial value and (3) the chain force acts along the end-to-end vector. The value of $D_{2}$ is 1.566 . If both factors are correct, then the discontinuity in the modulus at zero strain should be the product of the two, 2.12. As the first factor, $D_{1}$, suffices to account for the modulus discontinuity observed in experiment, it appears that the second factor is unnecessary and must therefore be incorrect. Therefore, one or more of our assumptions for $D_{2}$ must be wrong. The most likely candidate is the assumption that the chain force acts along the end-to-end vector, an assumption also inherent to classical elasticity theory.

Additional measurements of the modulus discontinuity closer to zero strain will be necessary before we can reach a firm conclusion about the validity of this analysis. We note that the discontinuity factors that we have obtained assumed only that an affine transformation of the node coordinates occurs, and should be applicable to any network, regardless of the polymer type or crosslink density.

This work was performed under the auspices of Los Alamos National Laboratory, which is operated by Los Alamos National Security, LLC, for the National Nuclear Security Administration of the U.S. Department of Energy under contract DE-AC5206NA25396, and at the Naval Research Laboratory with support from the Office of Naval Research.

\section{REFERENCES AND NOTES}

1 Rubenstein, M.; Colby, R. H. Polymer Physics; Oxford University Press, Inc: Oxford, UK, 2003.

2 Kuhn, W.; Grun, F. Kolloiddzschr 1942, 101, 248.

3 James, H. M.; Guth, E. J Chem Phys 1943, 11, 455-481.

4 James, H. M.; Guth, E. J Chem Phys 1947, 15, 669-683.

5 James, H. M.; Guth, E. J Polym Sci Polym Phys Ed 1949, 4, 153-182.

6 Erman, B.; Flory, P. J. J Polym Sci Polym Phys Ed 1978, 16, 1115-1121.

7 Pak, H.; Flory, P. J. J Polym Sci Polym Phys Ed 1979, 17, 1845.

8 McKenna, G. G.; Zapas, L. J. Polymer 1983, 24, 1502.

9 Mott, P. H.; Roland, C. M. Macromolecules 1996, 29, 6941.

10 McKenna, G. B.; Zapas, L. J. Polymer 1983, 1495-1501.

11 McKenna, G. B.; Zapas, L. J. Rubber Chem Technol 1986, 130-137.

12 Van der Hoff, B. M. E.; Glynn, P. A. R. J Macromol Sci Chem A 1969, 3, 991-1004.

13 Blokland, R. Elasticity and Structure of Polyurethane Networks; Rotterdam University Press, Gordon and Breach: NewYork, 1968. 


\title{
Corrigendum: Theoretical Implications of the Elastic Modulus Discontinuity in Rubber Networks
}

\author{
David E. Hanson, ${ }^{1}$ C.M. Roland ${ }^{2}$ \\ ${ }^{1}$ Theoretical Division, Los Alamos National Laboratory, Los Alamos, New Mexico 87545 \\ ${ }^{2}$ Chemistry Division, Naval Research Laboratory, Washington, DC 20375-5342
}

Received 13 June 2014; accepted 26 June 2014; published online 11 July 2014

DOI: 10.1002/polb.23542

Published in the J. Polym. Sci. Part B: Polym. Phys. 2010, 48(16), 1795-1798; DOI: 10.1002/polb.22045

There are two errors with respect to Eq. 14, one mathematical and one having to do with the underlying physics (Hanson, D.E., Roland, C.M. Theoretical Implications of the Elastic Modulus Discontinuity in Rubber Networks. Journal of Polymer Science Part B: Polymer Physics 2010:48(16):1795-1798). The normalizing factor in $\left(\theta_{2}-\theta_{1}\right)$ is incorrect; it should be the definite integral of $\sin \theta$ with $\theta_{1}$ to $\theta_{2}$. More significant is an error in the physics where we calculated the average chain extension factor by integrating the extension factor $G(\theta)$, which, by Eq. 6, is seen to be with respect to the end-to-end distance $R-R_{0}$. We then incorrectly associated the average chain extension factor with the stress. The subsequent analysis is also incorrect. The correct approach would involve deriving an expression for the average the chain energy which would be proportional to the quantity $\left(R-R_{0}\right)^{2}$, but a rigorous exposition for this derivation is beyond the scope of this Corrigendum.

However, we still believe that the principal conclusion of the paper is valid, namely that there is a discontinuity in the stress modulus between tension and compression. We have examined the change in the stress modulus between tensile and compressive strains using a previously published numer- ical network simulation model (EPnet) ${ }^{1-4}$ having chain forces consistent with the assumptions in Eq. 12. In the region between $\pm 0.2 \%$ of zero strain, we predict a stress discontinuity factor of $0.63 \pm 0.017$ (compressive modulus divided by the tensile modulus).

\section{ACKNOWLEDGMENTS}

We wish to thank Dr. John Barber of The Los Alamos National Laboratory for making us aware of the errors in the original paper.

\section{REFERENCES AND NOTES}

1 D. E. Hanson, Journal of Chemical Physics 2000, 113(17), 7656-7662.

2 D. E. Hanson, Polymer 2004, 45 (3), 1058-1062.

3 D. E. Hanson, Journal of Chemical Physics 2009, 131, 224904 (224905 pp.).

4 D. E. Hanson and J. L. Barber, Modelling and Simulation in Materials Science and Engineering 2013, 21. 